\title{
2016 Year in Review: Mechanical Ventilation
}

\author{
Tommaso Pettenuzzo MD and Eddy Fan MD PhD
}

\author{
Introduction \\ Epidemiology, Quality, and Outcomes \\ The LUNG SAFE Study: Still Room For Improvement in the \\ Management of ARDS \\ Ventilator Bundle Components: Not Created Equal \\ Overnight Extubation: Nothing Good Happens at Night in the ICU \\ Ventilator-Associated Challenges \\ Noninvasive Ventilation: A Double-Edged Sword \\ Ventilator-Induced Diaphragm Dysfunction: The Force Awakens \\ Spontaneous Breathing During Mechanical Ventilation: Not Always \\ Good \\ Ultrasound-Guided Assessment of Diaphragmatic Dysfunction: A New \\ Hope \\ Driving Pressure During General Anesthesia: The Difference .... Makes \\ a Difference \\ Oxygen Therapy in the Critically Ill Patient: Too Much of a Good \\ Thing? \\ Ventilator Liberation and Weaning \\ Proportional Assist Ventilation: What Patients Want \\ Acetazolamide in Patients With COPD: There May Be Something There \\ Physical Therapy: How to Make the First Move \\ Summary
}

The fundamental goals of mechanical ventilation are to improve pulmonary gas exchange and relieve respiratory distress, thus permitting lung and airway healing, while at the same time lessening the risk for iatrogenic complications. This review will summarize some of the advances in mechanical ventilation in 2016, with a particular focus on ventilator-associated clinical challenges and outcomes. Key words: critical care; respiratory distress syndrome; adult; review; ventilation; artificial; ventilation-induced lung injury. [Respir Care 2017;62(5):629-635. (C) 2017 Daedalus Enterprises]

\section{Introduction}

The fundamental goals of mechanical ventilation are to improve pulmonary gas exchange and relieve respiratory

\footnotetext{
The authors are affiliated with the Interdepartmental Division of Critical Care Medicine, University of Toronto and the University Health Network, Toronto, Ontario, Canada.
}

The authors have disclosed no conflicts of interest. distress, thus permitting lung and airway healing, while at the same time lessening the risk for iatrogenic complications. ${ }^{1}$ Long after Andreas Vesalius' legendary pilgrimage

\footnotetext{
Correspondence: Eddy Fan MD PhD, Toronto General Hospital, 585 University Avenue, PMB 11-123, Toronto M5G 2N2, Ontario, Canada. E-mail: eddy.fan@uhn.ca.
}

DOI: $10.4187 /$ respcare. 05545 
to the Holy Land to atone for the sin of restarting a Spanish nobleman's heart by inflating his lungs, ${ }^{2}$ the application of mechanical ventilation in acute or chronic respiratory failure due to pulmonary or systemic insults became commonplace in the ICU. This review will summarize some of the advances in mechanical ventilation in 2016, with a particular focus on ventilator-associated clinical challenges and outcomes.

\section{Epidemiology, Quality, and Outcomes}

ARDS is a common cause of acute respiratory failure (ARF), which is the leading indication for the initiation of mechanical ventilation. ${ }^{3}$ However, limited information is available about the epidemiology, management, and outcomes of patients with the ARDS, especially after the publication of the latest diagnostic criteria, known as the Berlin Definition. ${ }^{4}$ Moreover, uncertainty still exists regarding the factors influencing the quality of care and outcomes of mechanically ventilated patients.

\section{The LUNG SAFE Study: Still Room for Improvement in the Management of ARDS}

The Large Observational Study to Understand the Global Impact of Severe Acute Respiratory Failure (LUNG SAFE) was an international, multi-center, prospective cohort study of subjects undergoing invasive or noninvasive ventilation, conducted during 4 consecutive weeks in the winter of 2014 in a convenience sample of 459 ICUs from 50 countries across 5 continents. ${ }^{5}$ The study focused on ARDS epidemiology and outcomes in a large international cohort, the application of ventilatory interventions and adjuncts in routine practice, and the factors associated with ARDS recognition and its effect on management.

First, ARDS remains a common and lethal form or respiratory failure. Of 29,144 subjects admitted to participating ICUs, $10.4 \%$ fulfilled ARDS criteria, accounting for $23.4 \%$ of mechanically ventilated subjects. Unadjusted ICU and hospital mortality from ARDS were 35.3 and $40.0 \%$, respectively, and they increased with ARDS severity, along with the duration of invasive mechanical ventilation and ICU stay. Of note, the study confirmed the predictive validity of the Berlin Definition ${ }^{4}$ and, in an exploratory analysis, the association between driving pressure and mortality. ${ }^{6}$

Second, ARDS appears to be under-recognized and undertreated. Only $60.2 \%$ of all subjects with ARDS (ranging from $51.3 \%$ for mild to $78.5 \%$ for severe ARDS) were recognized by clinicians, and diagnosis was frequently delayed. Furthermore, one third of the subjects in whom plateau pressure was reported did not receive lung-protective ventilatory strategies, and evidence-based adjuncts (eg, prone positioning) were used infrequently. Interestingly, physician recognition had just a limited effect on treatment decisions. Taken together, the results of the LUNG SAFE study suggest that there is substantial room for improvement in the quality of care being delivered to patients with ARDS around the world.

\section{Ventilator Bundle Components: Not Created Equal}

Ventilator bundles are groups of interventions that are increasingly being implemented in the ICU to improve outcomes for patients undergoing mechanical ventilation. The most consistently adopted measures are head-of-bed elevation, daily interruptions of sedative infusions, daily spontaneous breathing trials (SBTs), thromboembolism prophylaxis, stress ulcer prophylaxis, and oral care with chlorhexidine gluconate. In their retrospective, single-center study including 5,539 subjects who underwent mechanical ventilation for $\geq 3 \mathrm{~d}$, Klompas et $\mathrm{al}^{7}$ suggested that we should revisit the classic ventilator bundle, which advocates for the aforementioned processes of care by assigning them equal weight and grouping them into a common package. In fact, head-of-bed elevation, sedative infusion interruptions, SBTs, and thromboprophylaxis were all associated with shorter time to extubation, and sedative infusion interruptions and SBTs were associated with lower rates of ventilator mortality. Sedative infusion interruptions were also associated with shorter time to hospital discharge and lower hospital mortality. On the contrary, stress ulcer prophylaxis and oral care with chlorhexidine not only had no effect on the duration of mechanical ventilation but were associated with higher risk for ventilatorassociated pneumonia and ventilator mortality, respectively. Indeed, a potential optimization of ventilator bundles should maximize sedative infusion interruptions and SBTs and reconsider whether oral care protocols should exclude chlorhexidine oral care and reserve stress ulcer prophylaxis for patients at increased risk for upper gastrointestinal tract bleeding. The efficacy of a revised ventilator bundle should be evaluated in a rigorous clinical trial.

\section{Overnight Extubation: Nothing Good Happens at Night in the ICU}

The correct timing of extubation is another crucial aspect in the management of the critically ill patient. Considering the significant morbidity associated with mechanical ventilation, patients should be extubated as soon as they are clinically ready, irrespective of the time of day. In the first multi-center evaluation of overnight extubation in the United States, Gershengorn et $\mathrm{al}^{8}$ assessed the frequency and safety of this procedure in a cohort of 97,844 mechanically ventilated adults included in the Project IMPACT database. 
One fifth $(20.1 \%)$ of the subjects underwent overnight extubation (from 7:00 pm to 6:59 am), whose likelihood increased in ICUs with intensivists on-site overnight (compared with other clinicians) and decreased with ICU admission year. Overnight extubation was associated with higher ICU and hospital mortality, both in the cohort of subjects who had a mechanical ventilation duration of $<12$ h (ICU: $5.6 \%$ vs $4.6 \%, P=.03$; hospital: $8.3 \%$ vs $7.0 \%, P=.01)$ and in those with mechanical ventilation duration of at least $12 \mathrm{~h}$ (ICU: $11.2 \%$ vs $6.1 \%, P<.001$; hospital: $16.0 \%$ vs $11.1 \%, P<.001)$. The latter cohort also showed higher rates of re-intubation $(14.6 \%$ vs $12.4 \%$, $P<.001)$.

Conversely, overnight extubation was associated with a slightly shorter length of ICU stay in the group with mechanical ventilation duration $<12 \mathrm{~h}$, and particularly for surgical subjects, and this practice may even be beneficial to some patients not included in the study (eg, those who have had cardiac surgery). However, the reported association with mortality should raise serious concerns about the routine practice of overnight extubation in the critically ill patient. In conclusion, the quality of care and outcomes of patients undergoing mechanical ventilation might be further benefited by improved knowledge of the epidemiology and how the clinicians manage ARDS, reevaluation of the crucial components of the ventilator bundle, and awareness that elective extubations, like major planned events in the ICU, should take place during the day.

\section{Ventilator-Associated Challenges}

The rationale for endotracheal intubation and mechanical ventilation should be to optimize conditions for clinical recovery and not for the restoration of "normal" physiological values, which would probably be deleterious to patients. The paradigm of lung-protective mechanical ventilation has traditionally advocated for the least injurious ventilatory settings and adjunctive strategies, ${ }^{9}$ but increasing attention has also been placed on the potentially harmful effects of mechanical ventilation on the heart ${ }^{10}$ and the diaphragm. ${ }^{11}$ The recognition of these mechanisms of injury to alternative organs prompted the development of heart-protective (eg, right ventricle) and muscle-protective (eg, diaphragm) mechanical ventilation strategies. ${ }^{11}$

\section{Noninvasive Ventilation: A Double-Edged Sword}

In their review of current clinical challenges in mechanical ventilation for subjects with ARF, Goligher et al ${ }^{11}$ debate the concept of multimodal protective mechanical ventilation. Noninvasive ventilation (NIV) has proven highly effective to prevent endotracheal intubation in patients with exacerbations of COPD, obesity-hypoventila- tion syndrome, and acute decompensated heart failure. However, a definite beneficial effect of NIV in the heterogeneous group of patients admitted for hypoxemic ARF has still to be demonstrated. Possible reasons for that are the concomitant circulatory failure, which might require complete respiratory muscle rest, and the risk for large non-protective tidal volumes delivered by NIV. Moreover, the authors endorse the effectiveness of NIV in immunocompromised hypoxemic patients and highlight the potential of high-flow nasal cannula therapy for patients with ARF.

Nonetheless, regardless of the NIV modality or the clinical indication, the patient's gas exchange, work of breathing, and mental status should be accurately monitored because of the risk for delayed intubation to worsen clinical outcome. This topic was recently emphasized by Bellani et $\mathrm{al}^{12}$ in a LUNG SAFE substudy. Among the causes of acute hypoxemic respiratory failure, NIV was not uncommonly used in the management of the subject with ARDS $(15 \%)$, irrespective of the severity. Potential advantages of NIV in this setting are mainly related to the avoidance of complications related to sedation, muscle paralysis, and invasive mechanical ventilation. However, the subgroup of patients with ARDS most likely to benefit from NIV remains unclear, and the impact of NIV on outcome in ARDS is still not well understood. Additionally, although the mortality rate was low in subjects successfully managed with NIV, subjects who failed NIV had a high mortality. Notably, NIV may be associated with higher ICU mortality in patients with moderate or severe ARDS.

\section{Ventilator-Induced Diaphragm Dysfunction: The Force Awakens}

Ventilator-induced diaphragm dysfunction can originate from several factors associated with critical illness and mechanical ventilation. ${ }^{11}$ Diaphragm inactivity due to excessive ventilatory support may cause rapid diaphragm atrophy and contractile dysfunction. However, insufficient diaphragm unloading can result in excessive inspiratory effort, sarcomeric disruption, and contractile fatigue, with consequent patient distress and muscle injury. Finally, eccentric lengthening contractions during patient-ventilator asynchrony further heighten the risk for acute muscle injury and weakness.

Considering that ventilator-induced diaphragm dysfunction can impair the successful liberation from mechanical ventilation and recovery from critical illness and is associated with increased morbidity and mortality, muscleprotective ventilation strategies are needed with the aim of maintaining appropriate levels of inspiratory muscle effort and optimal patient-ventilator synchrony: (1) management of sedation and ventilatory settings to find a safe compromise between the benefits and risks of spontaneous breath- 
ing during mechanical ventilation; (2) alternative modes of ventilation, such as proportional assist ventilation (PAV) and neurally adjusted ventilatory assist, in which the patient determines the level of inspiratory pressure support; (3) adjunctive strategies (eg, phrenic nerve stimulation); and (4) respiratory muscle rehabilitation in patients with established diaphragm dysfunction. ${ }^{11}$

\section{Spontaneous Breathing During Mechanical Ventilation: Not Always Good}

The delicate balance between the unloading of patient's respiratory muscles and the maintenance of appropriate levels of inspiratory effort during mechanical ventilation in injured lungs was evaluated in an experimental study from Yoshida et al. ${ }^{13}$ Spontaneous breathing during mechanical ventilation may be associated with improvements in lung aeration, ventilation-perfusion ( $(\dot{\mathrm{V}} / \mathbf{Q})$ matching, and gas exchange, a smaller risk of diaphragm deconditioning, and a reduced need for sedation and paralysis. However, the potential increases in transpulmonary pressure associated with spontaneous breathing can generate or worsen lung stress and strain, especially when lung injury is more severe. Another mechanism of harmful inflation during spontaneous breathing in injured lungs is pendelluft, displacement of gas from nondependent more recruited regions to dependent less recruited regions during strong diaphragm contractions at early inspiration. Although pendelluft may improve gas exchange, it can cause regional volutrauma and may lead to transient recruitment of atelectatic lung (ie, tidal recruitment), thus potentially causing ventilator-induced lung injury. In this setting, higher PEEP may ameliorate the negative swings in pleural pressure generated by spontaneous breathing and decrease the propensity to pendelluft through the increase in the endexpiratory lung volume and the radius of curvature of the diaphragm.

To test their hypotheses, the authors assigned 7 pigs to 4 consecutive combinations of PEEP levels (optimized or low) and spontaneous breathing (present or absent) and assessed tidal recruitment with dynamic computed tomography and regional $\dot{V} / \dot{Q}$ using electrical impedance tomography. The findings of this study described a novel mechanism of injurious ventilation: spontaneous breathing in the context of lung de-recruitment (ie, low PEEP), despite

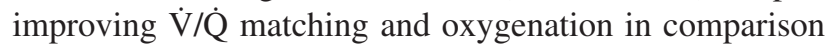
with muscle paralysis, caused larger pendelluft, which was proportional to tidal recruitment. However, optimization of lung recruitment with elevated PEEP reduced the intensity of the inspiratory efforts and minimized pendelluft, thereby protecting against ventilator-induced lung injury during spontaneous breathing.

Although these findings require confirmation in a clinical study, some important considerations for clinical prac- tice emerged from this study. First, we should avoid vigorous spontaneous breathing effort during mechanical ventilation in severe ARDS, because pendelluft may contribute to lung injury and mortality; this highlights a potential mechanism for benefit of early and short-term use of muscle paralysis in these patients. ${ }^{14}$ Second, the main benefits of spontaneous breathing can be achieved when sufficient PEEP is ensuring adequate lung recruitment. Third, spontaneous breathing could be introduced early at high PEEP levels, and weaning pressure support might take priority over weaning from PEEP during the process of mechanical ventilation liberation. Fourth, standard monitoring (eg, tracheal pressure and flow) cannot detect pendelluft; therefore, additional monitoring is important, such as electrical impedance tomography ${ }^{15}$ as well as measurements of the strength of spontaneous effort (eg, esophageal manometry, electromyography, ultrasound $)^{11}$ and transpulmonary driving pressure $\left(\Delta \mathrm{P}_{\mathrm{tp}}=\mathrm{P}_{\mathrm{tp}_{\mathrm{insp}}}-\mathrm{P}_{\mathrm{tp}_{\mathrm{exp}}}\right) .^{16}$

\section{Ultrasound-Guided Assessment of Diaphragmatic Dysfunction: A New Hope}

Many experts advocate the individualization of mechanical ventilation through the tailoring of ventilator settings to the patient's specific respiratory mechanics, such as inspiratory effort and static respiratory system compliance, ${ }^{11}$ although large randomized controlled trials (RCTs) testing ventilator strategies guided by these bedside measurement techniques are still lacking.

Monitoring of diaphragm activity has been advised to adjust the breathing work load in the individual patient to prevent muscle atrophy and fatigue..$^{17}$ Methods to assess diaphragmatic function are often difficult to implement in the ICU; however, bedside point-of-care ultrasound evaluation of diaphragm structure (ie, thickness) and function (ie, thickening) has proven to be feasible and accurate. ${ }^{18} \mathrm{In}$ the first study investigating the degree of diaphragm atrophy associated with different ventilation settings in critically ill patients, Zambon et al ${ }^{19}$ reported a linear relationship between the level of ventilation support and diaphragm thickening fraction and atrophy rate, thus confirming the clinical utility of diaphragm ultrasound in monitoring the time course of ventilator-induced diaphragm dysfunction and potentially evaluating muscle-protective mechanical ventilation strategies.

\section{Driving Pressure During General Anesthesia: The Difference ... Makes a Difference!}

Static respiratory system compliance is the change in lung volume for a given driving pressure and reflects the degree of lung volume loss due to atelectasis. By adjusting the tidal volume to respiratory system compliance, targeting driving pressure $(\Delta \mathrm{P}=$ plateau pressure $-\mathrm{PEEP})$ 
might better protect the lung in patients with more severe lung injury and low end-expiratory lung volume. Moreover, driving pressure adoption in setting the tidal volume may allow an "open" lung strategy in patients with larger end-expiratory lung volume, thus leading to a more precise titration of sedation. Driving pressure was associated with mortality in patients with ARDS, ${ }^{6}$ but its importance in patients with healthy lungs during surgery is unknown.

Neto et $\mathrm{al}^{20}$ performed an individual patient data metaanalysis from 17 RCTs (2,250 subjects) comparing protective ventilation with conventional ventilation in this setting. Driving pressure during intra-operative mechanical ventilation and a change in the level of PEEP resulting in an increase in driving pressure were found to be independently associated with the development of pulmonary complications after surgery. Additionally, driving pressure was reported to be the only significant mediator of the benefits of protective mechanical ventilation, which were less related in proportion to changes in tidal volume or level of PEEP. Although an RCT comparing mechanical ventilation based on driving pressure versus usual care is necessary to confirm these findings, this study shed light on how to provide protective mechanical ventilation during surgery. Notably, changes in the levels of PEEP leading to constant or increasing driving pressure are probably not resulting in lung recruitment but rather overstretching.

\section{Oxygen Therapy in the Critically Ill Patient: Too Much of a Good Thing?}

The potentially harmful role of direct oxygen toxicity in the critically ill patient should not be overlooked. Girardis et $\mathrm{al}^{21}$ investigated whether the application of a conservative protocol for oxygen supplementation could improve outcomes in comparison with a conservative protocol. Indeed, some evidence warns against the potential iatrogenic harm due to long periods of hyperoxia in the critically ill patient ${ }^{22,23}$ and supports the feasibility of a conservative oxygenation strategy in mechanical ventilation patients. ${ }^{24}$ The conservative protocol targeted $\mathrm{aP}_{\mathrm{aO}_{2}}$ of $70-100 \mathrm{~mm} \mathrm{Hg}$ or $\mathrm{S}_{\mathrm{pO}_{2}}$ of $94-98 \%$, whereas the conventional protocol targeted a $\mathrm{P}_{\mathrm{aO}_{2}}$ of up to $150 \mathrm{~mm} \mathrm{Hg}$ and an $\mathrm{S}_{\mathrm{pO}_{2}}$ of $97-$ $100 \%$. Subjects in the conservative group had significantly lower ICU mortality $(11.6 \%$ vs $20.2 \%, P=.01)$ and hospital mortality $(24.2 \%$ vs $33.9 \%, P=.03)$; lower risk for new bloodstream infections, shock, and liver failure; and more hours free from mechanical ventilation. Additionally, data revealed a U-shaped relationship between $\mathrm{P}_{a}$ values and mortality, with the highest mortality observed in subjects exposed to a $\mathrm{P}_{\mathrm{aO}_{2}}$ of $\geq 107 \mathrm{~mm} \mathrm{Hg}$.

Although the results of this trial were probably biased by its unplanned early termination due to difficulties with subject inclusion after a violent earthquake and small number of events, the evidence favoring the safety of a con- servative oxygen supplementation strategy supports the modern protective ventilation paradigm. Considering the potential multifaceted damage of mechanical ventilation, due to high airway pressures, excessive spontaneous ventilation, exaggerated diaphragm inactivity or fatigue, and toxic oxygen supplementation, the safest management of mechanically ventilated patients should be inspired by the principle that "less is more."

\section{Ventilator Liberation and Weaning}

Liberation of patients from mechanical ventilation depends on the synergistic coordination among the functions of the heart, lungs, respiratory and axial skeletal muscles, and brain. Several specific clinical problems can be responsible for an impairment in the weaning phase: (1) weaning-induced pulmonary edema; (2) weakness of the skeletal muscles, particularly respiratory muscles; (3) factors involving clinician behavior (eg, low use of routine SBTs); and (4) the possible accumulation of sedative drugs, also resulting in poor sleep quality, delirium, and longterm neurocognitive deficits (eg, benzodiazepines). Consequently, in patients who are difficult to liberate from mechanical ventilation, assessment of cardiovascular and diaphragmatic function should be obtained. ${ }^{11}$ Given the reduction in the duration of mechanical ventilation and ICU stay and a lower value on resource use reported by the available evidence, a recent clinical practice guideline suggested managing acutely hospitalized adults who have been mechanically ventilated for $>24 \mathrm{~h}$ with a ventilator liberation protocol. ${ }^{25}$

To maximize the safety and effectiveness of extubation, strategies aiming at the maintenance of a favorable balance between respiratory system capacity and load should be adopted, to stimulate adequate inspiratory effort and avoid diaphragm atrophy and patient-ventilator asynchrony. Different interventions are already available in daily clinical practice, such as the accurate titration of sedation and choice of sedative drugs, specific methods of ventilation designed to adjust the inspiratory support to the patient effort (eg, PAV and neurally adjusted ventilatory assist), pharmacologic adjuncts (eg, acetazolamide), and respiratory muscle rehabilitation. ${ }^{11}$

\section{Proportional Assist Ventilation: What Patients Want}

PAV is a mode of ventilation that measures patient respiratory demand and calculates compliance and resistance to offload the respiratory muscles in proportion to and in synchrony with patient effort. Previous studies of the effect of PAV during weaning from mechanical ventilation were limited in the number of patients and duration and most of them used an earlier version of PAV. Bosma et $\mathrm{al}^{26}$ compared the physiologic and clinical performance 
of a PAV with load-adjustable gain factors $(\mathrm{PAV}+)$ protocol with a pressure-support ventilation protocol for the entire duration of weaning to assess the feasibility of implementing these strategies in clinical practice. In their cohort of 50 subjects with difficult or prolonged weaning, protocols using progressive decreases in the level of assistance to find the lowest level of support tolerated without subject fatigue, coupled with daily assessment for SBTs, were proven to be used safely and effectively from the beginning of recovery until ventilation discontinuation. Additionally, PAV + was found to associate with less ventilator assistance relative to the pressure support group, thus suggesting that subjects receiving PAV + shared a greater portion of the total respiratory work load with the ventilator. Interestingly, the PAV + group was able to maintain the same minute ventilation with fewer episodes of respiratory distress and tended to have a lower asynchrony index. Finally, although there was no significant difference in time to extubation, this study reported a shorter time to ICU discharge in the PAV+ group, whose impact on outcome should be evaluated in a larger multi-center RCT.

\section{Acetazolamide in Patients With COPD: There May Be Something There}

In their multi-center RCT including 15 adult ICUs in France, Faisy et $\mathrm{al}^{27}$ hypothesized that acetazolamide could shorten the mechanical ventilation duration in critically ill patients suffering from exacerbations of COPD. Acetazolamide has been used for decades as a respiratory stimulant in this setting. In this study, subjects who were expected to receive mechanical ventilation for $>24 \mathrm{~h}$ were randomized to the high-dose acetazolamide $(500-1,000 \mathrm{mg}$, twice daily) or placebo group in cases of pure or mixed metabolic alkalosis. Although acetazolamide had no significant effect on duration of mechanical ventilation or weaning and respiratory parameters, the magnitude of the difference in duration of mechanical ventilation between the 2 groups was clinically important $(16 \mathrm{~h})$. Both statistical and pharmacologic factors were suggested to explain this negative finding, particularly the possibility that the study was underpowered to establish statistical significance.

\section{Physical Therapy: How to Make the First Move}

One of the most debilitating limitations for survivors of ARF is the long-term impairment in physical function due to prolonged mechanical ventilation and immobility, resulting in significant muscle wasting, immobility-related complications, and reduced post-ICU survival. Several observational studies report the feasibility and safety of early physical therapy programs; however, evidence from RCTs is still controversial. ${ }^{25}$
Moss et al ${ }^{28}$ conducted a 6-month follow-up RCT to test the hypothesis that an intensive physical therapy program was associated with significant improvements in long-term measures of physical functional performance in comparison with a standard-of-care physical therapy program. In their cohort of 120 critically ill subjects with ARF who required mechanical ventilation for $\geq 4 \mathrm{~d}$ and underwent a 28-d rehabilitation program, an intensive physical therapy program did not improve physical functional performance at any of the follow-up time points. This study confirmed that patients who survive ARF have severe and persistently diminished physical functioning and suggested that the implementation of an intensive physical therapy program may not be indicated for all of these patients. Indeed, future research studies are needed to identify the correct indication and dosage (timing and duration) of physical therapy and to risk stratify patients for specific components of physical therapy.

Similar results were reported by Morris et $\mathrm{al}^{29}$ in their RCT, which investigated the early delivery of standardized rehabilitation therapy through hospital discharge and its effect on hospital stay and long-term physical and mental function. In their cohort of 300 subjects with ARF, standardized rehabilitation compared with usual care did not decrease hospital stay, nor did it affect ventilator- or ICUfree days. Furthermore, function-related and health-related quality-of-life outcomes were similar for the 2 study groups at hospital discharge. Nonetheless, some of the physical function measurements evaluated in the study significantly improved in the standardized rehabilitation group at the 6-month follow-up. This suggested that the early rehabilitation program could have strengthened some components of physical function, thus inclining the standardized rehabilitation group to have greater movement while in the out-patient setting.

Although the overall available evidence is still limited, a recent clinical practice guideline, ${ }^{25}$ which reported on literature previous to the 2 studies just reviewed, suggested protocolized rehabilitation directed toward early mobilization for acutely hospitalized adults who have been mechanically ventilated for $>24 \mathrm{~h}$. The reason for this recommendation was the association between early physical therapy and the reduction in the duration of mechanical ventilation and increase in the likelihood of being able to walk at discharge, along with a lower value on cost and resource use.

\section{Summary}

Many important studies were published in 2016 that have improved our knowledge on epidemiology and outcomes of mechanically ventilated patients, as well as highlighting the main challenge of preventing iatrogenic harm from mechanical ventilation itself. The choice of the cor- 


\section{Year in Review: Mechanical Ventilation}

rect timing for the initiation and termination of the ventilatory support and greater use of lung- and muscle-protective strategies and interventions should be the target of clinicians in daily practice and the subject of ongoing and future research.

\section{REFERENCES}

1. Tobin MJ. Mechanical ventilation. N Engl J Med 1994;330(15): 1056-1061.

2. Colice GL. Historical perspective on the development of mechanical ventilation. In: Tobin MJ, editor. Principles and practice of mechanical ventilation, 3rd edition. New York: McGraw Hill Medical; 2012:3-41.

3. Esteban A, Anzueto A, Alía I, Gordo F, Apezteguía C, Pálizas F, et al. How is mechanical ventilation employed in the intensive care unit? An international utilization review. Am J Respir Crit Care Med 2000;161(5):1450-1458.

4. ARDS Definition Task Force, Ranieri VM, Rubenfeld GD, Thompson BT, Ferguson ND, Caldwell E, et al. Acute respiratory distress syndrome: the Berlin Definition. JAMA 2012;307(23):2526-2533.

5. Bellani G, Laffey JG, Pham T, Fan E, Brochard L, Esteban A, et al. Epidemiology, patterns of care, and mortality for patients with acute respiratory distress syndrome in intensive care units in 50 countries. JAMA 2016;315(8):788-800.

6. Amato MB, Meade MO, Slutsky AS, Brochard L, Costa EL, Schoenfeld DA, et al. Driving pressure and survival in the acute respiratory distress syndrome. N Engl J Med 2015;372(8):747-755.

7. Klompas M, Li L, Kleinman K, Szumita PM, Massaro AF. Associations between ventilator bundle components and outcomes. JAMA Intern Med 2016;176(9):1277-1283.

8. Gershengorn HB, Scales DC, Kramer A, Wunsch H. Association between overnight extubations and outcomes in the intensive care unit. JAMA Intern Med 2016;176(11):1651-1660.

9. The Acute Respiratory Distress Syndrome Network. Ventilation with lower tidal volumes as compared with traditional tidal volumes for acute lung injury and the acute respiratory distress syndrome. N Engl J Med 2000;342(18):1301-1308.

10. Repessé X, Charron C, Vieillard-Baron A. Acute cor pulmonale in ARDS: rationale for protecting the right ventricle. Chest 2015;147(1): 259-265.

11. Goligher EC, Ferguson ND, Brochard LJ. Clinical challenges in mechanical ventilation. Lancet 2016;387(10030):1856-1866.

12. Bellani G, Laffey JG, Pham T, Madotto F, Fan E, Brochard L, et al. Noninvasive ventilation of patients with acute respiratory distress syndrome: insights from the LUNG SAFE Study. Am J Respir Crit Care Med 2017;195(1):67-77.

13. Yoshida T, Roldan R, Beraldo MA, Torsani V, Gomes S, De Santis $\mathrm{RR}$, et al. Spontaneous effort during mechanical ventilation: maximal injury with less positive end-expiratory pressure. Crit Care Med 2016;44(8):e678-e688.

14. Papazian L, Forel JM, Gacouin A, Penot-Ragon C, Perrin G, Loundou A, et al. Neuromuscular blockers in early acute respiratory distress syndrome. N Engl J Med 2010;363(12):1107-1116.

15. Frerichs I, Amato MB, van Kaam AH, Tingay DG, Zhao Z, Grychtol B, et al. Chest electrical impedance tomography examination, data analysis, terminology, clinical use and recommendations: consensus statement of the TRanslational EIT developmeNt stuDy group. Tho$\operatorname{rax} 2017 ; 72(1): 83-93$.

16. Cortes GA, Marini JJ. Two steps forward in bedside monitoring of lung mechanics: transpulmonary pressure and lung volume. Crit Care 2013;17(2):219.

17. Bellani G, Pesenti A. Assessing effort and work of breathing. Curr Opin Crit Care 2014;20(3):352-358.

18. Zambon M, Greco M, Bocchino S, Cabrini L, Beccaria PF, Zangrillo A. Assessment of diaphragmatic dysfunction in the critically ill patient with ultrasound: a systematic review. Intensive Care Med 2017; 43(1):29-38.

19. Zambon M, Beccaria P, Matsuno J, Gemma M, Frati E, Colombo S, et al. Mechanical ventilation and diaphragmatic atrophy in critically ill patients: an ultrasound study. Crit Care Med 2016;44(7):13471352.

20. Neto AS, Hemmes SN, Barbas CS, Beiderlinden M, FernandezBustamante A, Futier E, et al. Association between driving pressure and development of postoperative pulmonary complications in patients undergoing mechanical ventilation for general anaesthesia: a meta-analysis of individual patient data. Lancet Respir Med 2016; 4(4):272-280.

21. Girardis M, Busani S, Damiani E, Donati A, Rinaldi L, Marudi A, et al. Effect of conservative vs conventional oxygen therapy on mortality among patients in an intensive care unit: the oxygen-ICU randomized clinical trial. JAMA 2016;316(15):1583-1589.

22. Wang $\mathrm{CH}$, Chang WT, Huang $\mathrm{CH}$, Tsai MS, Yu PH, Wang AY, et al. The effect of hyperoxia on survival following adult cardiac arrest: a systematic review and meta-analysis of observational studies. Resuscitation 2014;85(9):1142-1148.

23. Stub D, Smith K, Bernard S, Nehme Z, Stephenson M, Bray JE, et al. Air versus oxygen in ST-segment-elevation myocardial infarction. Circulation 2015;131(24):2143-2150.

24. Panwar R, Hardie M, Bellomo R, Barrot L, Eastwood GM, Young $\mathrm{PJ}$, et al. Conservative versus liberal oxygenation targets for mechanically ventilated patients: a pilot multicenter randomized controlled trial. Am J Respir Crit Care Med 2016;193(1):43-51.

25. Girard TD, Alhazzani W, Kress JP, Ouellette DR, Schmidt GA, Truwit JD, et al. An official American Thoracic Society/American College of Chest Physicians clinical practice guideline: liberation from mechanical ventilation in critically ill adults: rehabilitation protocols, ventilator liberation protocols, and cuff leak tests. Am J Respir Crit Care Med 2017;195(1):120-133.

26. Bosma KJ, Read BA, Bahrgard Nikoo MJ, Jones PM, Priestap FA, Lewis JF. A pilot randomized trial comparing weaning from mechanical ventilation on pressure support versus proportional assist ventilation. Crit Care Med 2016;44(6):1098-1108.

27. Faisy C, Meziani F, Planquette B, Clavel M, Gacouin A, Bornstain $\mathrm{C}$, et al. Effect of acetazolamide vs placebo on duration of invasive mechanical ventilation among patients with chronic obstructive pulmonary disease: a randomized clinical trial. JAMA 2016;315(5): 480-488.

28. Moss M, Nordon-Craft A, Malone D, Van Pelt D, Frankel SK, Warner ML, et al. A randomized trial of an intensive physical therapy program for patients with acute respiratory failure. Am J Respir Crit Care Med 2016;193(10):1101-1110.

29. Morris PE, Berry MJ, Files DC, Thompson JC, Hauser J, Flores L, et al. Standardized rehabilitation and hospital length of stay among patients with acute respiratory failure: a randomized clinical trial. JAMA 2016;315(24):2694-2702. 